\title{
Bm kettin, homologue of the Drosophila kettin gene, is located on the $Z$ chromosome in Bombyx mori and is not dosage compensated
}

\author{
MASATAKA G. SUZUKI†, TORU SHIMADA* \& MASAHIKO KOBAYASHI \\ Department of Agricultural and Environmental Biology, Graduate School of Agricultural and Life Sciences, \\ The University of Tokyo, Yayoi, Bunkyo-ku, Tokyo 113, J apan
}

In Bombyx mori, the female is the heterogametic sex and the sex determining system is referred to as ZZ/ZW. In a previous study, we found that this insect does not show dosage compensation at the transcriptional level. To confirm the validity of our conclusion, we investigated whether or not another sex-linked gene is dosage compensated. To identify new Z-linked genes, total RNA from reciprocal hybrid females between the silkworm strains p50 and C108 was compared using the differential display technique. Nine cDNA fragments corresponding to several differentially expressed mRNAs were cloned and sequenced. The analysis of nucleotide sequence polymorphisms confirmed that one of these cDNAs, ZDD4, originated from the $\mathrm{Z}$ chromosome. The amino acid sequence deduced from ZDD4 has homology with kettin, a modular protein in the Z-disc of Drosophila melanogaster muscles. On immunoblots of Bombyx larval muscle proteins a polypeptide of $380 \mathrm{kDa}$ was labelled with antibody to the ZDD4 peptide. We considered that the gene corresponding to ZDD4 encodes a kettin homologue in the silkworm, and denote it as Bm kettin. By a three-point cross, Bm kettin was mapped at $40.0 \mathrm{~cm}$ on the $\mathrm{Z}$ chromosome. Southern blot analysis revealed that Bm kettin was present at one copy in the genome. Northern blot analysis showed that Bm kettin mRNA was 9.1kb in length, and that the level of the mRNA in males was two times greater than that of females. Taken together with our previous observations, the present data suggest that lack of dosage compensation is a general rule in B. mori. Moreover, the twofold difference in Bm kettin expression between males and females may help explain the sexual difference in the wing flapping activity observed in some groups of Lepidoptera.

Keywords: Bombyx mori, dosage compensation, female heterogamety, kettin, sex-linked gene, $\mathrm{Z}$ chromosome.

\section{Introduction}

In Drosophila, orthopteran insects, mammals and Caenorhabditis elegans, the levels of X-linked gene expression are equalized between females (XX) and males $(\mathrm{XY})$ in order to compensate for the dosage effect of two X-linked alleles in females compared with one in males (Hebbert, 1984; Borsani \& Ballabio, 1993; Hsu \& Meyer, 1993; Baker et al., 1994). But it appears that dosage compensation is not an

*Correspondence. E-mail: shimada@ss.ab.a.u-tokyo.ac.jp $\dagger$ Present Address. Laboratory of Molecular Entomology and Baculovirology The Institute of Physical and Chemical Research (RIKEN), Hirosawa 2-1, Wako, Saitama 351-0198, Japan. absolute requirement even for animals with distinctly heteromorphic sex chromosomes. For example, in birds and butterflies, there is an apparent lack of dosage compensation for Z-linked genes (Stehr, 1959; Cook, 1964; Johnson \& Turner, 1979; Baverstock et al., 1982). In the domestic fowl, house sparrow and spotted turtledove, cytoplasmic aconitase is Z-linked and its activity in males is twice that in females (Baverstock et al., 1982). In Heliconius melpomene and $H$. elato, the activity of 6-phosphogluconate dehydrogenase (6PGD) located on the $\mathrm{Z}$ chromosome is not compensated between the two sexes (Johnson \& Turner, 1979). Data on several sex-linked morphological characteristics in 
birds are consistent with a dosage effect on Z-linked genes (Cook, 1964). Moreover, the $\mathrm{Z}$ chromosome does not show cytological indications of dosage compensation (such as enlargement of the single $\mathrm{X}$ in male Drosophila melanogaster or X-chromatin in female mammals) (Chandra, 1991).

These are only two reports that have confirmed the lack of dosage compensation by quantitatively comparing the gene expression level between males and females, and these are limited to the measurements of enzyme activity, as discussed above. Genes performing 'housekeeping' functions would be required to act throughout the life of an organism, and many such genes appear to be dose-insensitive, or largely so. For example, the copy number of genes coding for enzymes is not a significant factor in the control of metabolic flux (Kacser \& Burns, 1981). Similarly, heterozygotes for human metabolic mutations are not readily distinguishable from normal homozygotes because of a large overlap in enzyme levels. Such experience, and results of studies on the evolution of dominance, have led to the view that because of the structure of enzyme networks and the interactions among enzymes, their substrates, and products, 'a $50 \%$ reduction in activity, a common feature for many mutants, is not detectable in the phenotype' (Kacser \& Burns, 1981). Therefore, one can explain that housekeeping genes - such as those coding for enzymes - would not be dosage compensated because of their doseinsensitivity, but it is possible that other Z-linked genes might show dosage compensation in birds and butterflies.

To confirm that most or all Z-linked genes are not compensated, it is required to determine whether or not various kinds of genes which are involved in growth control, developmental programmes in many organisms, and formation of structures of tissues and organs, are compensated. As is the case with other Lepidoptera, Bombyx mori has female heterogamety (Hashimoto, 1933). In a previous study (Suzuki et al., 1998), we revealed that one of the Z-linked genes in this species is not dosage compensated. In this paper, we performed the differential display technique in order to identify Z-linked genes exhaustively in the silkworm. This resulted in the successful isolation of another Z-linked gene which was considered to encode a homologue of Drosophila kettin, which is a large modular protein in the Z-disc of insect muscles.

We describe here the comparison of the kettin homologue (denoted as 'Bm kettin') mRNA level between males and females and discuss the implications of our results for a causative role for the lack of dosage compensation in the development of the sexual dimorphism in phenotype.

\section{Materials and methods}

\section{Animals}

Silkworm strain 914 (os e) and the $\mathrm{F}_{1}$ hybrid females resulting from the reciprocal cross between strains p50 $\left(++^{o s}+{ }^{e}\right)$ and C108 $\left(+^{o s}+{ }^{e}\right)$ were used. Strain 914 was denoted by the National Institute of Sericultural and Entomological Sciences, Tsukuba, Japan. p50 and C108 were originally supplied by the Institute of Genetic Resources, Faculty of Agriculture, Kyushu University, Fukuoka, Japan and the National Institute of Genetics, Mishima, Japan, respectively, and had been inbred for more than 10 generations. Larvae were reared on fresh mulberry leaves.

\section{Differential display of mRNA}

The genes that show sequence polymorphisms among the reciprocal hybrid females between strains p50 and C108 (CP: C108 female $\times$ p50 male $\mathrm{F}_{1}$ female; PC: 550 female $\times \mathrm{C} 108$ male $\mathrm{F}_{1}$ female) will be derived from genes located on the sex chromosomes or plasmagene. Therefore, mRNA extracted from midguts of PC and CP larvae were compared using the differential display technique in order to identify Z-linked genes in the silkworm.

Total RNA was isolated by the acid guanidinium thiocyanate phenol-chloroform (AGPC) method (Chomcyzynski \& Sacchi, 1987). Two $\mu \mathrm{g}$ of total RNA was reverse transcribed using an oligo (dT) primer (5'-CATTATGCTGAGGATATCTTTTTTTTTVV-3') and subsequently amplified by PCR with two sets of arbitrary primers (set 1: P6 (5'ATTAACCCTCACTAAATGCTGGGGA-3'), P10 (5' -ATTAACCCTCACTAAATGTGGGTCC-3'), and P11 (5'-ATTAACCCTCACTAAATGTGGGAGC-3'); set 2: P3 (5'-ATTAACCCTCACTAAATGCTGGTGG-3') and P4 (5'-ATTAACCCTCACTAAATGCTGGTAG-3')) using a Delta RNA Fingerprinting Kit (Clontech Laboratories, Palo Alto, CA) according to the protocol supplied by the manufacturer.

The amplified products were resolved by electrophoresis on $8 \mathrm{~m}$ urea, 5\% polyacrylamide DNA sequencing gels. For detection on a FluorImager SI (Molecular Dynamics, CA), following the removal of the upper glass plate, the gel was stained with Vistra Green (Molecular Dynamics, CA) according to the manufacturer's instructions. The stained gel was put 
on the sample tray and scanned at the high sensitivity mode.

\section{Cloning and DNA sequencing}

Differentially amplified cDNA fragments were excised from the gel, PCR reamplified and cloned into plasmid pGEM-T using the TA cloning kit from Promega (Madison, WI). Both strands of the cDNA fragments were sequenced with the ABI Prism Dye Terminator Cycle Sequencing Ready Reaction Kit (Perkin Elmer, Applied Biosystems Division, Foster City, CA) using M13-21 and RV primers. Some sequences were determined with the aid of synthetic primers.

\section{Sequence analysis}

Sequence data were analysed with the sequence analysis software package, DNASIS version 3 (Hitachi Software Engineering Co., Yokohama, Japan). The deduced amino acid sequence was compared with protein databases (Swiss-Prot and PIR) using the program BLASTP (Altschul et al., 1990). Amino acid sequences were aligned and compared with the aid of the CLUSTAL V program (Higgins et al., 1992).

\section{Electrophoresis and immunoblotting}

Longitudinal ventral muscles were dissected from fifth-instar larvae. The muscles were washed and then stored in rigor solution $(0.1 \mathrm{M} \mathrm{KCl}, 20 \mathrm{~mm}$ K-phosphate buffer $\mathrm{pH} 6.8,5 \mathrm{~mm} \mathrm{MgCl}_{2}, 5 \mathrm{~mm}$ EGTA, $5 \mathrm{~mm} \mathrm{NaN}_{3}$ ) with $50 \%$ glycerol at $-20^{\circ} \mathrm{C}$ as described by Bullard et al. (1985). The muscles were homogenized in rigor solution with $0.1 \%$ Triton $\mathrm{X}-100$ and $0.5 \mathrm{~mm}$-methylsulphonyl fluoride (PMSF), then washed in the same solution once, three times in rigor solution with $0.5 \mathrm{~mm}$ PMSF and twice in $5 \mathrm{~mm}$ Tris, $\mathrm{pH}$ 7.0. The muscle extract was heated in the SDS sample buffer as described by Wang (1982). Proteins were electrophoresed in SDS-polyacrylamide slab gels with the Laemmli (1970) buffer system and subsequently transferred to nitrocellulose filters by the method of Wang et al. (1989).

A polyclonal antibody against the ZDD4 peptide (Bmket2) was made by immunizing a rabbit with a bovine serum albumin-conjugated synthetic peptide representing residues 79-102 in Fig. 2. Immunodetection was performed with Bmket2 and goat antirabbit IgG conjugated with horseradish peroxidase using the ECL Western blotting system according to the manufacturer's instructions (Amersham International, Buckinghamshire, U.K.).

\section{Southern blot analysis}

Genomic DNA was extracted from the posterior silk glands of mid-fifth-instar larvae according to the protocol described previously (Suzuki et al., 1998). Digested DNAs were separated by electrophoresis on a $1.0 \%$ agarose gel and transferred to a Hybond$\mathrm{N}+$ nylon membrane (Amersham International, Buckinghamshire, U.K.). Blotted DNAs were hybridized with a ${ }^{32} \mathrm{P}$-labelled $1.0 \mathrm{~kb}$ HindIII fragment of ZDD4 which had been prepared by random primer labelling. Hybridization and washing were performed according to the manufacturer's instructions. Exposure, analysis and printing were performed using a BAS2000 Bioimage Analyser (Fuji Photo Film, Tokyo, Japan).

\section{Northern blot analysis}

Poly (A) + RNA was isolated from adult thoraces using a poly (A) + RNA extraction kit Quick Prep Micro (Pharmacia Biotech, Uppsala, Sweden) according to the protocol provided by the manufacturer. RNA was electrophoresed on $1.2 \%$ agarose- $2.2 \mathrm{M}$ formamide gels in MOPS buffer and transferred onto a Hybond-N + nylon membrane (Amersham International, Buckinghamshire, U.K.). The filter was then hybridized and washed following the procedures described previously (Suzuki et al., 1998). The radioactivity was visualized and quantified using a BAS 2000 Bioimage Analyser (Fuji Photo Film, Tokyo, Japan). As a control probe to ensure equal RNA loading, the actin1 gene of B. mori was used. The actin1 gene was synthesized by PCR amplification with primers BAF1 (5'-CGATGTCGTTGCGTTGTAG-3') and BAR4 (5'-GGCCGGACTCGTCGTATTCC-3') which were designed from the genomic DNA sequence (Mounier et al., 1987), and cloned as described above.

\section{Results}

Isolation of a Z-linked gene by the differential display technique

mRNA populations from single PC and CP larvae were compared by differential display. Eleven differentially amplified cDNA fragments (ZDD1, ZDD2, ZDD3, ..., ZDD11) were reamplified. Nine of them were successfully cloned (pGZD3, pGZD4, pGZD5,

(C) The Genetical Society of Great Britain, Heredity, 82, 170-179. 
..., pGZD11) and sequenced. To clarify which cDNA fragment was derived from a Z-linked gene, $\mathrm{Z}$ chromosome linkage analysis was performed by taking advantage of restriction fragment length polymorphisms (RFLPs) between strains p50 and C108. We found that the ZDD4 fragment amplified from p50 genomic DNA using PCR primers ZD43 (5'-TCTAGCTTCCTCAGCTTGTTC-3') and ZD44 (5'-TGGGTGAAGCTGTATCAACTG-3') was cleaved into $350 \mathrm{bp}$ and $173 \mathrm{bp}$ fragments with $\mathrm{DraI}$ (Fig. 1, lane 1), but that from C108 was not digested (Fig. 1, lane 2). The patterns of these RFLPs were different between males and females (Fig. 1, lanes 4 and 5). Moreover, the RFLPs differ in lanes 3 and 5 as expected for a Z-linked gene (Fig. 1, lanes 3 and 5). These results confirm that ZDD4 is a cDNA originated from a Z-linked gene in the silkworm. One of the other differentially amplified cDNAs was derived from the mitochondrial DNA and the remaining ones originated from autosomal genes. Hence, we decided to analyse ZDD4 in detail.

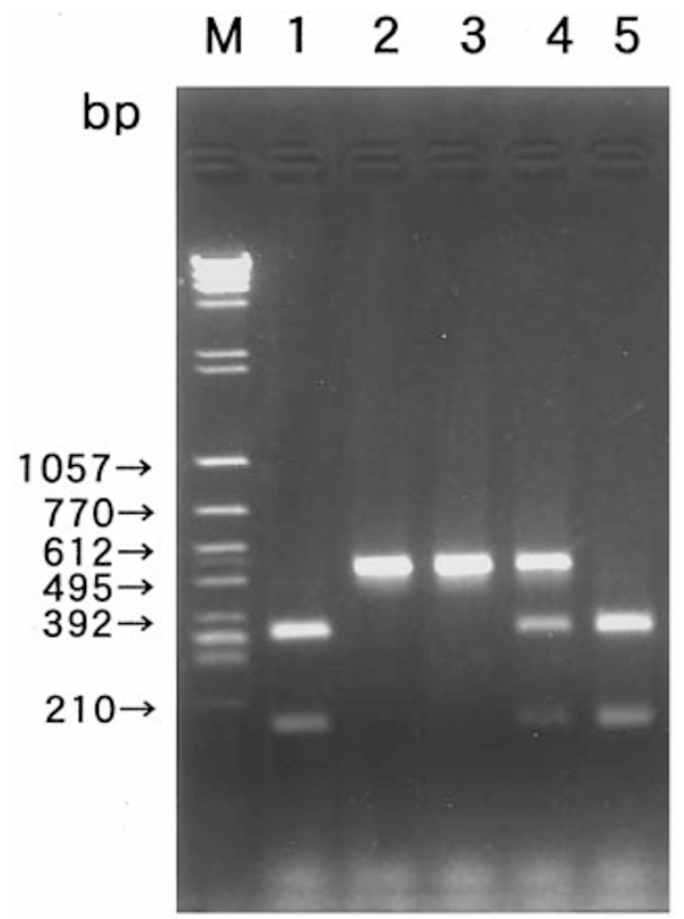

Fig. 1 Band patterns of PCR-RFLPs among the silkworm strains $\mathrm{p} 50, \mathrm{C} 108$ and their $\mathrm{F}_{1}$ individuals. PCR products amplified using ZD43 and ZD44 were digested with DraI and electrophoresed on a $2 \%$ agarose gel. $\mathrm{M}$, size marker; 1, p50; 2, C108; 3, p50 × C108 $\mathrm{F}_{1}$ female; 4, p50 $\times \mathrm{C} 108 \mathrm{~F}_{1}$ male; $5, \mathrm{C} 108 \times \mathrm{p} 50 \mathrm{~F}_{1}$ female.

\section{ZDD4 structure and sequence analysis}

The entire sequence of ZDD4 was determined and a 1405 bp sequence was found to correspond to a part of one open reading frame (ORF) (Fig. 2). A database search indicated that the deduced amino acid sequence of ZDD4 shared homology with muscle proteins, including Drosophila kettin, connectin in chicken, and titin in human. An alignment of the ZDD4 sequence with the partial sequence of Drosophila kettin is shown in Fig. 3. The kettin sequence consists of four repeats of class II (immunoglobulinC2-like) domains separated by insertions of 35 amino acids of unique sequence (Lakey et al., 1993). As shown in Fig. 3, it appeared that the ZDD4 sequence has a similar primary structure to kettin; that is, this peptide consists of four repeat units of 99 or 100 amino acids which are homologous to class II domains, separated by insertions of 32-46 amino acids that are not significantly homologous to any sequence in the database. Identical residues in kettin match well with identical residues in ZDD4: 19 positions in ZDD4 and kettin are identical. There is more homology between class II domains of the ZDD4 sequence (34 identical residues) than there is between class II domains of kettin (27 identical residues); notably 11 amino acids DSGIYTCKAYN (amino acids numbers 126-136, 258-268, 396-406 in Fig. 2) are invariant throughout the class II domains in ZDD4. In addition, the linker sequences between class II domains in ZDD4 are homologous to each other, with four identical residues.

These observations strongly suggest that ZDD4 is a part of the gene which encodes a high molecular weight muscle protein like kettin.

\section{Identification of the protein encoded by ZDD4}

To identify the protein which contains the amino acid sequence deduced from the nucleotide sequence of ZDD4, a polyclonal antiserum against the ZDD4 peptide was made by immunizing a rabbit with a synthetic peptide representing residues 79-102 in Fig. 2. On Western blots of an extract from longitudinal ventral muscles of fifth-instar larvae, this antiserum recognized a protein of $\approx 380 \mathrm{kDa}$ (Fig. 4), slightly smaller than Drosophila kettin $(500 \mathrm{kDa})$. The size of kettin mRNA is $11.5 \mathrm{~kb}$ (Lakey et al., 1993), whereas ZDD4 mRNA is $9.1 \mathrm{~kb}$ in length (Fig. 8). Therefore, the molecular weight of the protein encoded by the gene which contains ZDD4 is calculated as $500 \times 9.1 / 11.5=396 \mathrm{kDa}$.

These results suggest that the gene which contains ZDD4 apparently encodes a high molecular weight 
muscle protein whose function would be very similar to that of kettin. We have therefore called this gene 'Bm kettin'.

\section{Mapping of Bm kettin}

We further characterized Bm kettin by mapping its chromosomal location by means of a three-point cross using os (sex-linked translucent, 1-0.0) and $e$ (elongate, 1-36.4) as markers (Doira et al., 1992). Strains C108 $\left(+{ }^{o s}+{ }^{e}\right)$ and 914 (os $e$ ) were used in this experiment, and primers ZD43 and ZD44 were used for amplification. We found, as previously, that ZDD4 amplified from genomic DNA of C108 was not digested with $\mathrm{DraI}$, but that from 914 was cleaved into $350 \mathrm{bp}$ and $173 \mathrm{bp}$ fragments (Fig. 5). We denote the C108 type of ZDD4 as Bm kettin ${ }^{c}$ and the 914 type as Bm kettin $^{P}$. The mating scheme was as follows: $\mathrm{Bm}^{\mathrm{kettin}^{P}}$ os $e$ female $\times(\mathrm{Bm}$ kettin${ }^{c}+{ }^{o s}+{ }^{e}$ female $\times B m$ kettin ${ }^{P}$ os $e$ male) male. Numbers of individuals scored in the next generation are shown in Table 1 . Recombination values were calculated as $31.6 \%$ between os and $e, 32.7 \%$ between $o s$ and Bm kettin, and 3.1\% between $e$ and $B m$ kettin. Because the distance between os and $e$ has been determined to be $36.4 \mathrm{cM}$ (Doira et al., 1992), we calculated the locus of Bm kettin as $36.4+\{3.1 \times(36.4 / 31.6)\}=40.0 \mathrm{cM}$ (Fig. 6). Set (Soft and elongated trunk, 1-35.5), $e$ (elongate, 1-36.4), $V g$ (Vestigial, 1-38.7) and Bt (Beet feeder, 1-40.8) have been localized near this locus.

\section{Copy number of Bm kettin}

To compare the mRNA level by Northern blot hybridization between males and females, it is
1 CCCTCACTAAATGCTGGTGGCACAGTTTCTTCGACATCACTTAAAGTCAAAACTAAATCTACTATTATC 69 $\begin{array}{lllllllllllllllllllllllllllllll}1 & P & S & L & N & A & G & G & T & V & S & S & T & S & L & K & V & K & T & K & S & T & I & I & 23\end{array}$ 70 GACCAGGCCATGAGACCCGAGTCGTGGGAACAAATTCAATTAAAGGAAGCTGCCATGAACAAGGTTCCC 138 $\begin{array}{llllllllllllllllllllllllll}24 & \mathrm{D} & Q & A & M & R & P & E & S & W & E & Q & I & Q & L & K & E & A & A & M & N & K & V & P & 46\end{array}$ 139 GAAATGTTCGTTGACACTGGTGTTCAACAAGCCCCTATTTTCACTACACATCTAAAAAGCTACGACAAG 207 $\begin{array}{lllllllllllllllllllllllll}47 & E & M & F & V & D & T & G & V & Q & Q & A & P & I & F & T & T & H & L & K & S & Y & D & K & 69\end{array}$ 208 CTTGTAGAAGGACAGCACGTGTATTTGGAAGCTCAGGTAGAACCACGTACTGATCCAAACCTTAAGATT 276

$\begin{array}{lllllllllllllllllllllllll}70 & L & V & E & G & Q & H & V & Y & L & E & A & Q & V & E & P & R & T & D & P & N & L & K & I & 92\end{array}$ 277 GAGTGGTTCAAAAACGGAGTCACGCTAACTACTGGAGCTCGATTGAAGAGTACCTTGACTTTGGTTTAT 345

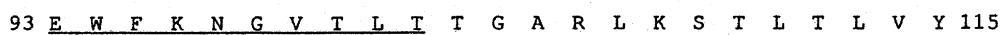
346 TACCTTTCTATCAATAGCTTACGAAATGATGATTCGGGTATTTACACATGTAAAGCAACAAATCTTTTG 414 $\begin{array}{llllllllllllllllllllllllllllll}116 & \mathrm{Y} & \mathrm{L} & \mathrm{S} & \mathrm{I} & \mathrm{N} & \mathrm{S} & \mathrm{L} & \mathrm{R} & \mathrm{N} & \mathrm{D} & \mathrm{D} & \mathrm{S} & \mathrm{G} & \mathrm{I} & \mathrm{Y} & \mathrm{T} & \mathrm{C} & \mathrm{K} & \mathrm{A} & \mathrm{T} & \mathrm{N} & \mathrm{L} & \mathrm{L} & 138\end{array}$ 415 GGTGAAGCTGTATCAACTGCATCGATTAAAATAGAAGATCGTCATTGGTTGCTTGGAGAATCACTTCAT 483 $\begin{array}{llllllllllllllllllllllllllll}139 & G & E & A & V & S & T & A & S & I & K & I & E & D & R & H & \text { W } & \text { L } & \text { L } & G & E & S & \text { L } & \text { H } & 161\end{array}$ 484 CCGGACTCACTCGATCGTATTGGGGCACTTGAGCAGCCCAAGCCTGAAAAACCTGAAGCTCCCGAACCA 552 $\begin{array}{lllllllllllllllllllllllll}162 & P & D & S & L & D & R & I & G & A & L & E & Q & P & K & P & E & K & P & E & A & P & E & P & 184\end{array}$ 553 ACTTATGAAACGCCCGTATTCATATCCCATTTAAATAATATTGTATGTAAAGAAGGAGATAATATTCAT 621 $\begin{array}{lllllllllllllllllllllllllll}185 & \mathrm{~T} & \mathrm{Y} & \mathrm{E} & \mathrm{T} & \mathrm{P} & \mathrm{V} & \mathrm{F} & \mathrm{I} & \mathrm{S} & \mathrm{H} & \mathrm{L} & \mathrm{N} & \mathrm{N} & \mathrm{I} & \mathrm{V} & \mathrm{C} & \mathrm{K} & \mathrm{E} & \mathrm{G} & \mathrm{D} & \mathrm{N} & \mathrm{I} & \mathrm{H} & 207\end{array}$ 622 TTCGAATGTAATGTGGAACCATCTAGAGATCCTACTCTTAAAATCGAGTGGTTCTTTAATAATAAACCT 690 $\begin{array}{llllllllllllllllllllllllll}208 & F & E & C & N & V & E & P & S & R & D & P & T & L & K & I & E & W & F & F & N & N & K & P & 230\end{array}$ 691 CTACCATCGGGAACTAGGTATAAGAGTACTCATGACTTCAGCTATGTTTCGCTTGATATAACGCATACT 759 $\begin{array}{llllllllllllllllllllllllllllll}231 & \text { L } & P & S & G & T & R & Y & K & S & T & H & D & F & S & Y & V & S & L & D & I & T & H & T & 253\end{array}$ 760 TACGAAGAGGATTCCGGTATTTACACTTGTAAAGCAACGAACAGCAAGGGCTCAGCTACAACTTCTGGT 828 $\begin{array}{lllllllllllllllllllllllll}254 & \text { Y } & E & E & D & S & G & I & Y & T & C & K & A & T & N & S & K & G & S & A & T & T & S & G & 276\end{array}$ 829 TCATTAAGATGCACGGGTGACAAAAACATTTACTTTGATACTCAGCATCCTCAAGGTAAAGCTGGTTTG 897 $\begin{array}{llllllllllllllllllllllllll}277 & \text { S } & \text { L } & \text { R } & \text { C } & \text { T } & G & D & K & \text { N } & \text { I } & \text { Y } & \text { F } & \text { D } & \text { T } & Q & \text { H } & \text { P } & Q & \text { G } & \text { K } & \text { A } & \text { G } & \text { L } & 299\end{array}$ 898 GAAGCTGTCGAACAAGCTGAGGAAGCTAGATTGGCAAAGGGTAGGAGGCCTTCCGTTCCGGATACAGGA 966

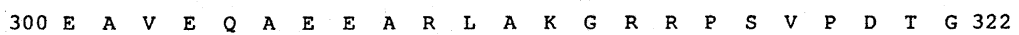
967 TATCCAAAACCAGAATGGATTATACCAATTGCTGCTGAGCATCAGCTAGTAGAAGGCCAAGTACTTCAT 1035 $\begin{array}{lllllllllllllllllllllllll}323 & \text { Y } & P & K & P & E & \text { W } & I & I & P & I & A & A & E & H & Q & \text { L } & V & E & G & Q & \text { V } & \text { L } & \text { H } & 345\end{array}$ 1036 TTGGAAGGCCAAGTAGAACCCAAGAATGATCCAGATCTCAAGATTGAGTGGTACTTCAATGGCAAAGTT 1104 $\begin{array}{llllllllllllllllllllllllll}346 & \text { L } & E & G & Q & V & E & P & K & N & D & P & D & \text { L } & K & I & E & W & Y & F & N & G & K & V & 368\end{array}$ 1105 TTAGAACAAGGTTCCAGATTTAAACTTACTAGTGATTTCGGATTCGTGACGCTAGATCTCGIGGACGTG 1173 $\begin{array}{lllllllllllllllllllllllllllllll}369 & \text { L } & E & \text { E } & Q & G & S & R & F & K & \text { L } & T & S & D & F & G & F & V & T & L & D & L & V & D & V & 391\end{array}$ 1174 TATGAAAGAGATAGTGGCATTTATACGTGCAAAGCTTACAACAAGAGGGGTGAAGCTTTTACTTCATCT 1242 $\begin{array}{llllllllllllllllllllllllll}392 & Y & E & R & D & S & G & I & Y & \text { T } & C & K & A & Y & N & K & R & G & E & A & F & T & S & S & 414\end{array}$ 1243 ACTGTATATTGCACTAGTAAAGATAATCTAATTGAAAGAACTCAACATCCGAAAGGAACTGAAGGTTTA 1311 $\begin{array}{lllllllllllllllllllllllll}415 & \mathrm{~T} & \mathrm{~V} & \mathrm{Y} & \mathrm{C} & \mathrm{T} & \mathrm{S} & \mathrm{K} & \mathrm{D} & \mathrm{N} & \mathrm{L} & \mathrm{I} & \mathrm{E} & \mathrm{R} & \mathrm{T} & \mathrm{Q} & \mathrm{H} & \mathrm{P} & \mathrm{K} & \mathrm{G} & \mathrm{T} & \mathrm{E} & \mathrm{G} & \mathrm{L} & 437\end{array}$

1312 GAAAAGATTCAAAATTTGGAGGTTCTCTTCGCAAAGAACCCGGGCAACAGCCTGATGACGATACAGGTA 1380 $\begin{array}{llllllllllllllllllllllllll}438 & \text { E } & \text { K } & \text { I } & Q & N & \text { L } & \text { E } & \text { V } & \text { L } & \text { F } & \text { A } & \text { K } & \text { N } & \text { P } & \text { G } & \text { N } & \text { S } & \text { L } & \text { M } & \text { T } & I & Q & \text { V } & 460\end{array}$ 1381 GACCACCAGCATTTAGTGAGGGTTA 1405 $\begin{array}{llllllllllllllllll}461 & D & H & O & H & \text { L } & V & R & \text { V } & 468\end{array}$
Fig. 2 The nucleotide sequence of ZDD4. The deduced amino acid sequence is shown below the nucleotide sequence. The amino acid sequence of the synthetic peptide for preparing the polyclonal antibody against the ZDD4 peptide is underlined. 

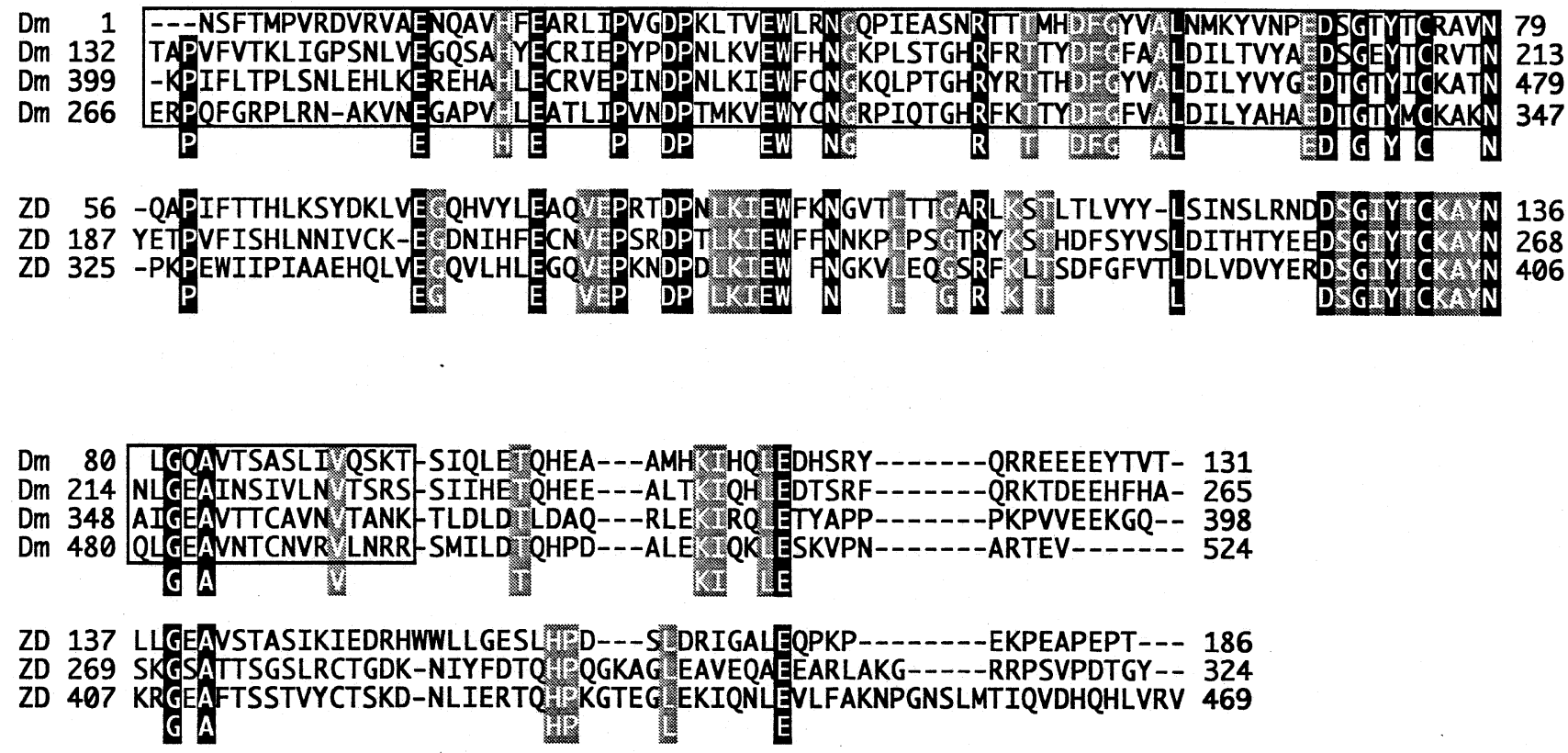

Fig. 3 Comparison between Drosophila kettin and Bombyx ZDD4. The rows indicated by Dm are the partial amino acid sequence of Drosophila kettin. The putative amino acid sequence of Bombyx ZDD4 is shown in the rows labelled ZD. The large boxes show the kettin class II domain from Lakey et al. (1993). Conserved amino acids are indicated below each block. Amino acids that are conserved between Drosophila kettin and Bombyx ZDD4 are black-boxed. Identical amino acids among the four repeats in Drosophila kettin and the three repeats in Bombyx ZDD4 are shaded.

required to confirm whether or not there are genes that share sequence similarity with $\mathrm{Bm}$ kettin. Genomic DNA from larval silk glands was digested with HindIII and blotted after gel electrophoresis. The Southern blot also included DNA from plasmid pGZD4 digested with HindIII, containing ZDD4 equivalent to $1,2,4$ and 8 copies per haploid male genome (i.e. one set of autosomes and one $\mathrm{Z}$ chromosome). When the Southern blot was probed with a ${ }^{32} \mathrm{P}$-labelled $1.0 \mathrm{~kb}$ HindIII fragment of ZDD4, the only genomic DNA band that hybridized was $1.0 \mathrm{~kb}$ in length (Fig. 7). When the intensities of the hybridization in the genomic DNAs are compared with those of the various equivalents in the cloned DNA, it is apparent that the copy number of ZDD4 is two in the male genome but one in the female genome. This result indicates that both males and females have one copy of the Bm kettin gene per $\mathrm{Z}$ chromosome.

Comparison of Bm kettin mRNA level between males and females

As shown above, Bm kettin is a single-copy gene located on the $\mathrm{Z}$ chromosome of B. mori. This suggests that $B m$ kettin can be used to investigate the dosage compensation of Z-linked genes in the silkworm by comparing the transcriptional level between males and females.

We examined the steady-state level of Bm kettin mRNA transcripts in the two sexes by Northern blot hybridization. Poly (A) + RNA from adult thoraces was subjected to Northern blot analysis using ZDD4 as a probe. One major mRNA species of $9.1 \mathrm{~kb}$, slightly smaller than Drosophila kettin mRNA (11.5kb), was observed (Fig. 8). The probe also hybridized to a number of different mRNA species of various sizes less than $9.1 \mathrm{~kb}$. Because Bm kettin is a single-copy gene, it seems therefore that the broad and smeared signal was caused by the degradation of the mRNA because of its great length.

In the present experiment, we considered the $9.1 \mathrm{~kb}$ mRNA to be a major transcript of Bm kettin, and compared the level of this mRNA between males and females. As shown in Table 2, the level of Bm kettin mRNA transcript in male RNA was 1.8 times greater than that of the female RNA. To determine whether the observed difference in the steady-state levels of Bm kettin mRNAs in males and females is a result of inaccuracy in RNA quantification, the steady-state level of Bombyx actin1 mRNA was also measured in these two RNA samples. As 
shown in Table 1, the ratio of the steady-state level of $B m$ kettin mRNA relative to actin1 mRNA in males was approximately twice that in females.

This result indicates that there is an apparent lack of dosage compensation for $\mathrm{Bm}$ kettin in the silkworm.

\section{Discussion}

In this report, we revealed that a single copy Z-linked gene which is considered to be a kettin homologue in the silkworm was not dosage compensated. Taken together with our previous study, which confirmed that a novel Z-linked gene, T15, is not compensated, our findings suggest that lack of dosage compensation is a general rule in the silkworm.

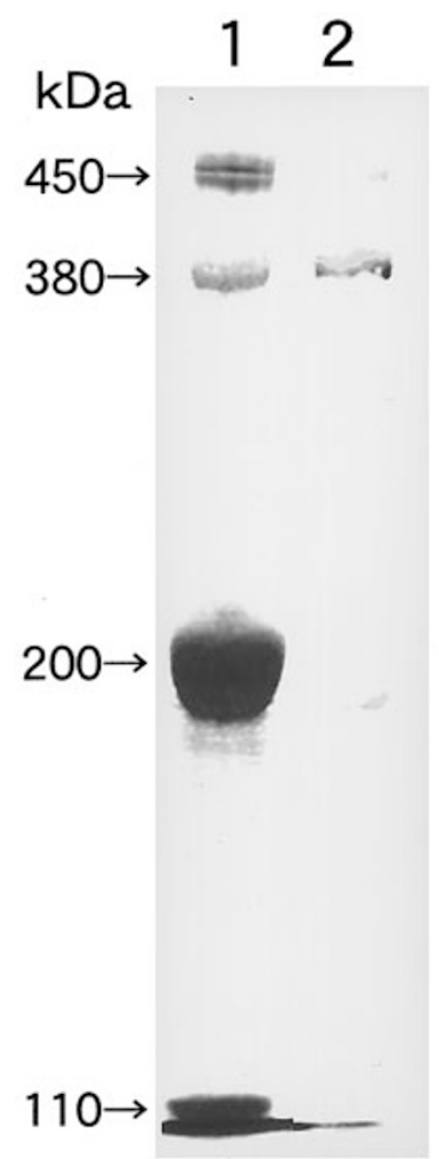

Fig. 4 Identification of the protein encoded by ZDD4 in Bombyx mori. Lane 1, SDS-polyacrylamide gel (4\% acrylamide) of longitudinal ventral muscles of Bombyx fifthinstar larvae. Proteins are detected by Coomassie blue stain. Lane 2, immunoblots incubated with polyclonal antibody Bmket2.

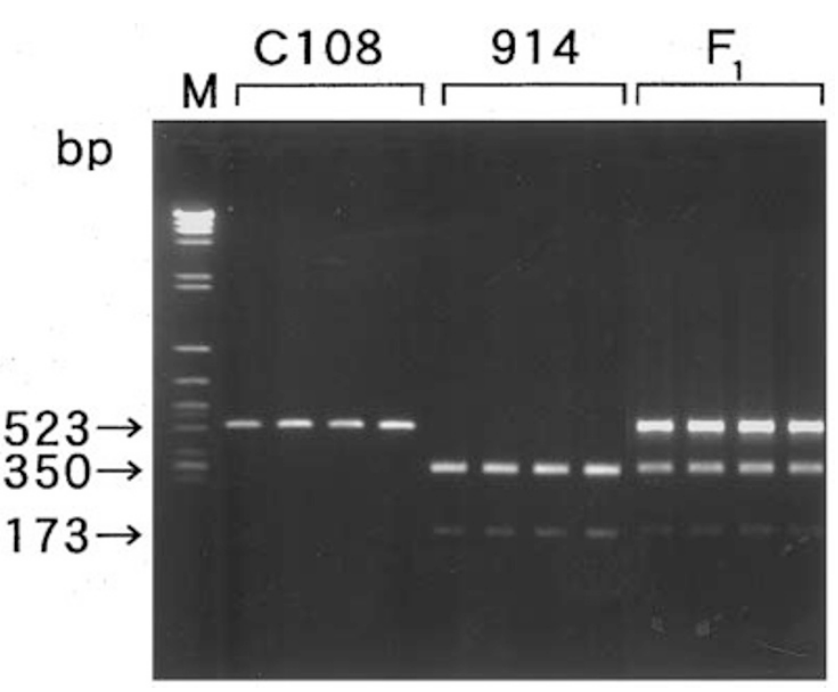

Fig. $5 \mathrm{Bm}$ Kettin PCR-RFLP between the silkworm strains C108 and 914. PCR products amplified from genomic DNA of strains C108 (C108), 914 (914) and individuals from the $F_{1}$ hybrid generation between a C108 female and a 914 male $\left(\mathrm{F}_{1}\right)$ using the primers ZD43 and ZD44 were digested with DraI and separated on a $2.0 \%$ agarose gel. $\mathrm{M}$, size marker.

It has been noted that in species known to have dosage compensation (Drosophila, orthopteran insects, mammals and Caenorhabditis elegans), the males are the heterogametic sex; in contrast, in groups lacking dosage compensation (birds, butter-

Table 1 Three-point cross among Bm kettin, os and $e$

\begin{tabular}{|c|c|c|}
\hline \multirow[b]{2}{*}{ Phenotypes } & \multicolumn{2}{|c|}{ No. of individuals } \\
\hline & Male & Female \\
\hline Bm kettin $^{P}+{ }^{o s}+{ }^{e}$ & 0 & 0 \\
\hline$B m$ kettin $^{P / C}+{ }^{o s}+{ }^{e}$ & 17 & 0 \\
\hline Bm kettin ${ }^{C}+{ }^{o s}+{ }^{e}$ & 0 & 17 \\
\hline Bm kettin ${ }^{P}$ os e & 16 & 15 \\
\hline Bm kettin ${ }^{P / C}$ os e & 2 & 0 \\
\hline Bm kettin ${ }^{C}$ os e & 0 & 0 \\
\hline $\mathrm{Bm} \mathrm{kettin}^{P}+{ }^{o s} e$ & 9 & 7 \\
\hline Bm kettin ${ }^{P / C}+{ }^{o s} e$ & 0 & 0 \\
\hline Bm kettin ${ }^{C}+{ }^{o s} e$ & 0 & 1 \\
\hline Bm kettin $^{P}$ os $+^{e}$ & 0 & 0 \\
\hline Bm kettin ${ }^{P / C}$ os $+^{e}$ & 7 & 0 \\
\hline Bm kettin ${ }^{C}$ os $+^{e}$ & 0 & 7 \\
\hline Total & 51 & 47 \\
\hline \multicolumn{3}{|c|}{$\begin{array}{l}\text { The individuals in the } \mathrm{F}_{2} \text { hybrid generation between the } \\
\text { silkworm strain } 108 \times 914 \text { were used. Bm kettin } \\
\text { represents the individuals that have triple bands of the } \\
\text { C-type } B m \text { kettin sequence (see Fig. } 5 \text { ) }\end{array}$} \\
\hline
\end{tabular}


flies and the silkworm), females are heterogametic. In birds, the $\mathrm{Z}$ chromosome, like the $\mathrm{X}$, is one of the larger chromosomes and makes up nearly $10 \%$ of the haploid genome (Ohno, 1967). Therefore, the viability of heterogametic females in spite of an absence of dosage compensation raises questions about the role of this process.

Several studies on butterflies suggest that the absence of dosage compensation may be the result of a precise adaptation (Stehr, 1959; Grula \& Taylor, 1980; Sperling, 1994). Johnson \& Turner (1979) suggested that the absence of dosage compensation may provide the metabolic basis for the limitation of expression to the female sex, which is a common feature of mimicry, and of some nonmimetic polymorphisms in butterflies. They also pointed out that the lack of dosage compensation would explain that female-limited characters will be predominantly autosomal, whereas male-limited sexual characters can be both sex-linked and autosomal (Johnson \& Turner, 1979). Among butterflies, a large proportion of genes controlling female mateselection behaviour and male courtship signals are located on the $\mathrm{Z}$ chromosome (Grula \& Taylor,

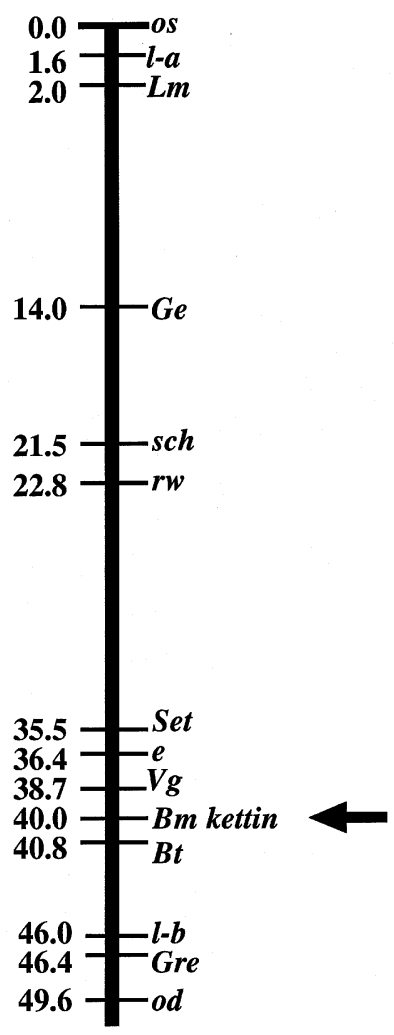

Fig. 6 Location of the Bm kettin locus on chromosome 1 ( = Z chromosome) of Bombyx mori. See text for symbols.
1980), and genetic data on these phenotypes are consistent with the view that most, if not all, of the $\mathrm{Z}$ chromosome lacks dosage compensation (Johnson \& Turner, 1979; Grula \& Taylor, 1980). Thus, in species which do not show dosage compensation, it appears that there is a close relationship between an absence of dosage compensation of Z-linked genes and the sexual dimorphism in phenotype.

$\mathrm{Bm}$ kettin is considered to be a homologue of Drosophila kettin, which is a modular protein in the $\mathrm{Z}$-disc of insect muscles. Muscle, in addition to the contractile and regulatory proteins, contains an important class of proteins that maintain the structural integrity of the sarcomere and may also be involved in morphogenesis. The Z-disk of striated muscle anchors the ends of thin filaments from neighbouring sarcomeres and has an ordered lattice structure that is hexagonal in insect flight muscle and tetragonal in vertebrates. Lakey et al. (1993) indicated that kettin is an important structural

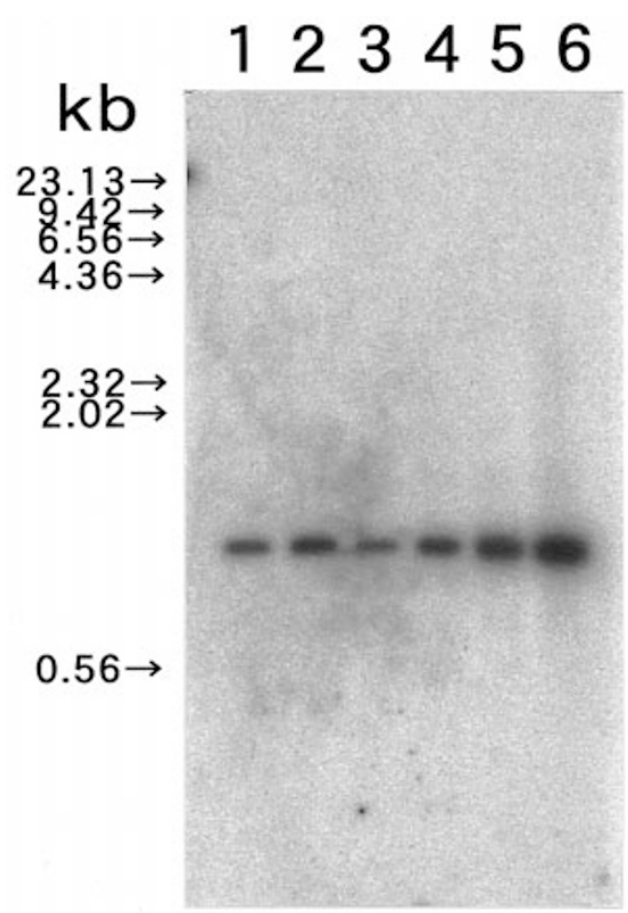

Fig. 7 Determination of the ZDD4 copy number in male and female genomic DNA of Bombyx mori. Genomic DNA from female (lane 1) and male (lane 2) larvae was digested with HindIII and electrophoresed in a $1.0 \%$ agarose gel. Included in the gel was DNA from plasmid pGZD4 digested with HindIII, 1, 2, 4 and 8 male haploid genome equivalents (lanes 3-6, respectively). A Southern blot of the gel was hybridized with a radiolabelled $1.0 \mathrm{~kb}$ HindIII fragment of ZDD4. Molecular size in $\mathrm{kb}$ is indicated to the left of the figure. 
component of the flight muscle Z-disk and acts as a scaffolding.

It appears that most genes that contribute to multicomponent structures such as ribosomes, spliceosomes and the muscle, in which stoichiometric ratios of individual components may be important, show dose-dependent effects later in development (Kongswan et al., 1985; Last et al., 1987; Homyk \& Emerson, 1988; Regan \& Fuller, 1988). The twofold difference in the Bm kettin mRNA level between males and females is probably responsible for the difference in the amount of the Bm kettin protein in the two sexes, and leads to the sexual difference, for example, in the muscular strength. In some groups of moths, males need to fly towards females, whereas females do not fly but wait for males and

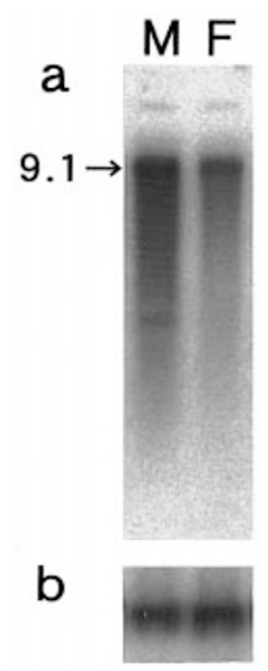

Fig. 8 Determination of the relative steady-state level of the Bm kettin mRNA in male and female poly $(\mathrm{A})+$ RNA extracted from adult thoraces of Bombyx mori. (a) Northern blots were hybridized with ${ }^{32} \mathrm{P}$-labelled ZDD4. M, male; F, female. (b) The same blot was hybridized with Bombyx actin1 DNA as a control. The molecular size in $\mathrm{kb}$ is indicated to the left of the figure. attract them by releasing sex pheromone. In extreme cases, such as some species of moths belonging to Geometridae and Psychidae, females cannot fly because of their very small and degraded wings (Inoue et al., 1982). Although adults of both sexes of $B$. mori are unable to fly, males flap their wings vigorously when they display courtship behaviour to females. In contrast to males, females scarcely flap their wings throughout their adult life, although sometimes wing fanning is observed during oviposition and calling behaviour.

The twofold difference in the Bm kettin mRNA level between males and females may be the result of an adaptation to the sexual difference in the demand for wing flapping activity as described above. Our observations suggest that the lack of dosage compensation for Z-linked genes could provide an opportunity for the evolutionary development of sexual dimorphism in phenotype.

\section{Acknow ledgements}

We are grateful to Dr M. R. Goldsmith for her helpful comments on the manuscript. We thank Sawady Technology Co. Ltd. for their assistance in the differential display. This work was supported in part by Grants-in-Aid for Scientific Research from the Ministry of Education, Science, Sports and Culture, Japan.

\section{References}

ALtSCHUl, S. F., GISH, W., MILleR, W., MYERS, E. W. AND LIPMAN, D. J. 1990. Basic local alignment search tool. J. Mol. Biol., 215, 403-410.

BAKER, B. S., GORMAN, M. AND MARIN, I. 1994. Dosage compensation in Drosophila. Ann. Rev. Genet., 28, 491-521.

BAVERSTOCK, P. R., ADAMS, M., POLKINGHORNE, R. W. AND GELDER, M. 1982. A sex-linked enzyme in birds -

Table 2 Levels of Bm kettin and Actin1 mRNA in male and female Bombyx mori

\begin{tabular}{|c|c|c|c|c|c|c|c|c|c|c|}
\hline \multirow[b]{2}{*}{ Probe } & \multicolumn{6}{|c|}{ PSL value $\dagger$} & \multicolumn{4}{|c|}{ Bm kettin/Actin1 ratio } \\
\hline & Male & $\mathrm{SE} \ddagger$ & $N$ & Female & $\mathrm{SE}$ & $N$ & Male & $\mathrm{SE}$ & Female & SE \\
\hline Bm kettin & 159.28 & 10.85 & 6 & 91.7 & 4.28 & 6 & 0.37 & 0.03 & 0.21 & 0.01 \\
\hline Actin1 & 434.16 & 15.90 & 6 & 437.34 & 9.70 & 6 & - & - & - & - \\
\hline
\end{tabular}

$\dagger$ PSL value indicates the mean radioactivity of six respective areas of Northern blots showing hybridization measured using a Bioimage Analyser BAS 2000.

$\ddagger \mathrm{SE}$ is the standard error of six separate determinations. 
Z-chromosome conservation but no dosage compensation. Nature, 296, 763-766.

BORSANI, G. AND BAllabio, A. 1993. X chromosome dosage compensation in female mammals. Semin. Dev. Biol., 4, 129-139.

BUllard, B., BELl, J., CRAIG, R. AND LEONARD, K. 1985. A new actin-like protein in insect flight muscle. J. Mol, Biol., 183, 443-454.

CHANDRA, H. S. 1991. How do heterogametic females survive without gene dosage compensation? J. Genet., 70, 137-146.

CHOMCYZYNSKI, P. AND SACCHI, N. 1987. Single-step method of RNA isolation by acid guanidinium thiocyanate-phenol-chloroform extraction. Analyt. Biochem., 162, 156-159.

COOK, A. G. 1964. Dosage compensation and sex-chromatin in non-mammals. Genet. Res., 5, 354-365.

DOIRA, H., FJII, H., KAWAGUCHI, Y., KIHARA, H. AND BANNO, Y. 1992. Genetical Stocks and Mutations of Bombyx mori: Important Genetic Resources. Institute of Genetic Resources, Faculty of Agriculture, Kyushu University.

GRULA, J. W. AND TAYLOR, O. R., JR. 1980. The effect of $\mathrm{X}$-chromosome inheritance on mate selection behaviour in the sulfur butterflies, Colias eurytheme Colias philodice. Evolution, 34, 688-695.

HAshimoto, н. 1933. The role of the $\mathrm{W}$ chromosome for sex determination in the silkworm, Bombyx mori. Jap. J. Genet., 8, 245-258.

HEBBERT, D. R. 1984. Dosage compensation of the sex-linked enzyme phosphoglucomutase in the Orthoptera. Heredity, 53, 361-369.

HIGGINS, D. G., BLEASBY, A. J. AND FUCHS, R. 1992. CLUSTAL-V: Improved software for multiple sequence alignment. Comput. Appl. Biosci., 8, 189-191.

HOMYK, T., JR. AND EMERSON, C. P., JR. 1988. Functional interactions between unlinked muscle genes within haploinsufficient regions of the Drosophila genome. Genetics, 119, 105-121.

HSU, D. R. AND MEYER, B. J. 1993. X chromosome dosage compensation and its relationship to sex determination in C. elegans. Semin. Dev. Biol., 4, 93-106.

INOUE, H., SUGI, S., KUROKO, H., MORIUCHI, S. AND KAWABE, A. 1982. Moths of Japan, vols 1 and 2. Kodansha, Tokyo.
JOHNSON, M. S. AND TURNER, J. R. G. 1979. Absence of dosage compensation for a sex-linked enzyme in butterflies (Heliconius). Heredity, 43, 71-77.

KACSER, H. AND BURNS, J. A. 1981. The molecular basis of dominance. Genetics, 97, 639-666.

KONGSWAN, K., YU, Q., VINCENT, A., FRISARDI, M. C., ROSBASH, M., LENGYEL, J. A. AND MERRIAM, J. 1985. A Drosophila Minute gene encodes a ribosomal protein. Nature, 317, 555-558.

LAEMMLI, U. K. 1970. Cleavage of structural proteins during the assembly of the head of bacteriophage T4. Nature, 227, 680-685.

LAKEY, A., LABEIT, S., GAUTEL, M., FERGUSON, C., BARlow, D. P., LEONARD, K. AND BULlaRD, B. 1993. Kettin, a large modular protein in the Z-disk of insect muscles. EMBO J., 12, 2863-2871.

LAST, R. L., MARDOCK, J. R. AND WOOLFORD, J. L., JR. 1987. Evidence for related functions of the RNA genes of Saccharomyces cerevisiae. Genetics, 117, 619-631.

MOUNIER, N., GAILlARD, J. AND PRUDHOMME, J. C. 1987. Nucleotide sequence of the coding region of two actin genes in Bombyx mori. Nucl. Acids Res., 15, 2781.

oHno, s. 1967. Sex-Chromosomes and Sex Linked Genes. Springer-Verlag, Berlin.

REGAN, C. L. AND FULLER, M. T. 1988. Interacting genes that affect microtubule function: the $n c 2$ allele of the haywire locus fails to complement mutations in the testis-specific b-tubulin gene of Drosophila. Genes Devel., 2, 82-92.

SPERLING, F. A. H. 1994. Sex-linked genes and species differences in Lepidoptera. Can. Ent., 126, 807-818.

STEHR, G. 1959. Haemolymph polymorphism in a moth and the nature of sex-controlled inheritance. Evolution, 13, 537-560.

SUZUKI, G. M., SHIMADA, T. AND KOBAYASHI, M. 1998. Absence of dosage compensation at the transcription level of a sex-linked gene in a female heterogametic insect, Bombyx mori. Heredity, 81, 275-283.

WANG, K. 1982. Purification of Titin and Neubulin. Methods Enzymol., 85, 264-274.

WANG, K., FANGER, B. O., GUYER, C. A. AND STAROS, J. V. 1989. Electrophoretic transfer of high-molecular-weight proteins for immunostaining. Methods Enzymol., 172, 687-696. 